\title{
Favorable long-term outcomes of one-year adjuvant S-1 monotherapy for pathological stage II or III gastric cancer treated at a high-volume center
}

\author{
Yosuke Kano ${ }^{1} \cdot$ Manabu Ohashi $^{1}$ (D) Naoki Hiki ${ }^{1} \cdot$ Daisuke Takahari $^{2} \cdot K^{2}$ isho Chin $^{2} \cdot K^{2}$ Kensei Yamaguchi ${ }^{2}$. \\ Yasuo Tsuda ${ }^{1}$. Yoshiaki Shoji ${ }^{1}$ - Itaru Yasufuku ${ }^{1} \cdot$ Kojiro Eto $^{1} \cdot$ Satoshi Ida $^{1} \cdot$ Koshi Kumagai $^{1} \cdot$ Souya Nunobe $^{1}$. \\ Takeshi Sano ${ }^{1}$
}

Received: 2 March 2018 / Accepted: 17 April 2018 / Published online: 26 April 2018

(c) The International Gastric Cancer Association and The Japanese Gastric Cancer Association 2018

\begin{abstract}
Background One-year adjuvant S-1 monotherapy following D2 gastrectomy has been the Japanese treatment standard for pathological stage II or III gastric cancer since the Adjuvant Chemotherapy Trial of S-1 for Gastric Cancer (ACTS-GC) was concluded in 2007. Trial patients were selected according to the 13th edition of the Japanese classification (JC-13). The JC-13 and the TNM classification underwent major revisions in 2010 (JC-14/TNM-7). However, neither the recent therapeutic results for patients with stage II/III disease defined by the current system nor comparisons with the ACTS-GCresults have been reported.

Methods The 390 study patients had pathological stage II/III gastric cancer defined by the JC-14/TNM-7 and treated with S-1 following D2 gastrectomy between 2008 and 2012. The completion rate of 1-year S-1, first relapse site, and stagespecific survival according to the JC-14/TNM-7, JC-13, and TNM-6 were examined and the results compared with those of the ACTS-GC.

Results The completion rate for 1-year S-1 (69.5\%) was slightly higher than in the ACTS-GC. The recurrence pattern was almost identical. The 5-year overall survival rates of pathological IIA, IIB, IIIA, IIIB, and IIIC in the JC-14/TNM-7 were $96.0,85.5,81.8,72.0$, and $51.1 \%$, respectively. Their 5-year overall and relapse-free survival rates by the JC-13 and TNM-6 systems were favorable as compared to those of ACTS-GC patients for all substages.

Conclusions Survival outcome shown in this study of patients treated with 1-year adjuvant S-1 after D2 gastrectomy at a high-volume cancer hospital will provide a reference for future adjuvant trials targeting JC-14/TNM-7 stage II/III disease.
\end{abstract}

Keywords Gastric cancer $\cdot$ S-1 $\cdot$ Adjuvant chemotherapy $\cdot$ ACTS-GC

\section{Introduction}

Gastric cancer is the third leading cause of cancer-related death in the world [1]. Gastrectomy is the principal treatment, with chemo- or chemoradiotherapy added to improve

Manabu Ohashi

manabu.ohashi@jfcr.or.jp

1 Department of Gastroenterological Surgery, Gastroenterological Center, Cancer Institute Hospital, Japanese Foundation for Cancer Research, 3-8-31 Ariake, Koto-ku, Tokyo 135-8550, Japan

2 Department of Gastroenterological Medicine, Gastroenterological Center, Cancer Institute Hospital, Japanese Foundation for Cancer Research, Tokyo, Japan the survival of patients with locally advanced tumors. Although neo-adjuvant chemotherapy is increasingly used in Western countries [2], primary surgery followed by adjuvant chemotherapy is still the mainstream therapeutic strategy in Japan [3], where many tumors are detected at a relatively early stage and thus can be cured by surgery alone or in a surgery-first approach.

The Adjuvant Chemotherapy Trial of S-1 for Gastric Cancer (ACTS-GC) was the first pivotal trial to show a significant survival benefit of adjuvant chemotherapy $[4,5]$. The more than 1000 patients enrolled in the trial were treated at 109 Japanese hospitals, including ours, after strict eligibility screening for intraoperative negative peritoneal cytology, complete D2 lymphadenectomy, and pathological confirmation of stage II or stage III disease. After publication of the 
trial's results, in 2007, showing a $10 \%$ higher 5 -year survival rate in the adjuvant group than in the surgery group, 1-year S-1 administration became the standard treatment for patients with pathologically confirmed stage II or III gastric cancer in Japan [3].

In the subsequent 10 years, extensive experience with this therapy has accumulated nationwide, but survival outcomes have not yet been reported or compared with those determined by the ACTS-GC. One of the problems is the major change in the gastric cancer staging system that took place after the ACTS-GC. Until the 13th edition of the Japanese Gastric Cancer Association (JGCA) staging system (JC-13), published in 1999, the extent of lymph node metastasis was assessed based on the anatomic location of the metastatic nodes $[6,7]$. The TNM system of the American Joint Committee on Cancer (AJCC) and the International Union for Cancer Control (UICC) had a similar, anatomically based nodal staging system until the 4th edition (TNM-4) [8, 9], but beginning with TNM-5, published in 1997, a numerical system was substituted in which nodal metastasis was graded by the number of metastatic nodes $[10,11]$. The ACTS-GC was conducted using the JC-13 system and the trial concluded in 2007. In 2010, the JGCA abandoned the traditional nodal grouping method and adopted a numerically based system (JC-14) together with major revisions of the AJCC/ UICC (TNM-7). JC-14 and TNM-7 employ the same, new definitions of $\mathrm{T}$ - and $\mathrm{N}$-categories and stage groups that are widely in use elsewhere in the world [12-15]. Thus, the current pathological stage II or III is not the same as it was in the ACTS-GC, especially with respect to the subgroups of these stages.

To date, the stage-specific survival outcomes of patients who received S-1 adjuvant therapy have yet to be evaluated according to the current staging system. In addition, the survival of ACTS-GC patients vs. those treated according to current practice has not been compared using the same staging system. As this information will be useful as a reference in future clinical trials, especially for those targeting more specific substages, we conducted a retrospective study at our high-volume specialized center analyzing patient survival according to different staging systems. We also compared our results with those in ACTS-GC by converting our data from JC-14 to JC-13.

\section{Patients and methods}

\section{Patients}

The data of patients who underwent R0 gastrectomy at the Cancer Institute Hospital, Tokyo, between January 2008 and December 2012 were retrospectively reviewed from our prospective database. Data recorded in the pathology section of the database include the location and depth of the primary tumor, the number and location of dissected lymph nodes, and the number and location of metastatic nodes. Thus, each tumor can be stage-grouped according to any staging system, including the JC-13, JC-14, TNM-6, or TNM-7 [16, 17]. Our study population consisted of 390 patients with pathological stage II or III gastric cancer, diagnosed based on the current staging system (JC-14/TNM-7). All of the patients satisfied the other eligibility criteria of the ACTS-GC and had received standard adjuvant S-1 monotherapy (Fig. 1). The median follow-up period was 60 months (range 5-96 months).

\section{Adjuvant therapy}

S-1 monotherapy was planned and administered according to the standard protocol of the ACTS-GC: $80-120 \mathrm{mg} \mathrm{S}-1 /$ day for 4 weeks, followed by 2 weeks of rest, to be continued either for a year after surgery or for eight courses. All patients were treated in the outpatient setting. Dose or schedule modifications of S-1 due to adverse events or the patient's condition were made mostly according to the standardized methods described in the ACTS-GC protocol [4].

In 2008 and 2009, S-1 was prescribed by either medical oncologists or surgeons, and thereafter only by medical oncologists. Patients visited the hospital every 2-6 weeks during S-1 therapy, then every 3 months during the following year, and every 6 months thereafter up to 5 years after surgery. They were evaluated based on the results of a physical examination, blood test, including the tumor markers CEA and CA19-9, and half-yearly abdominal computed

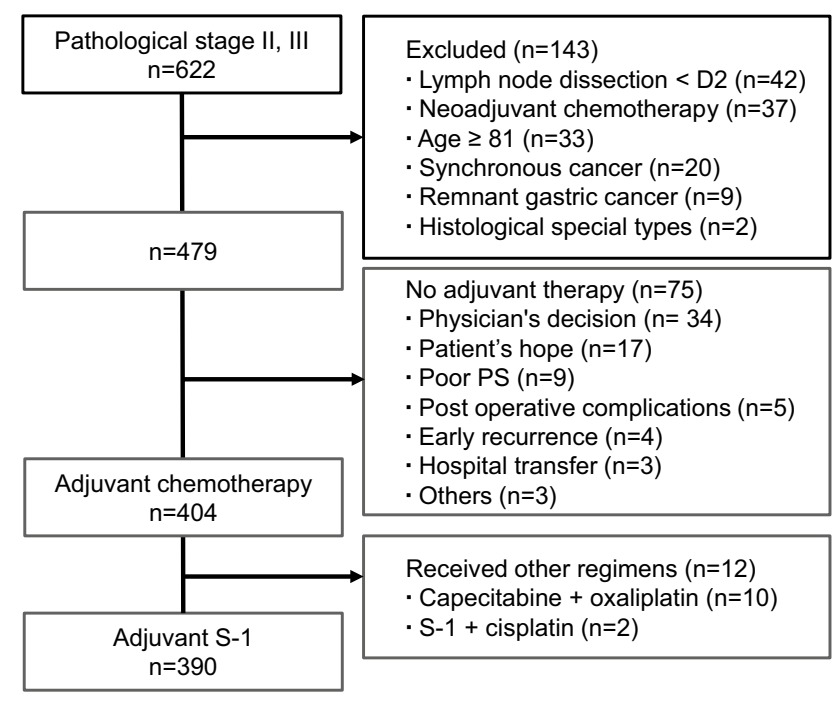

Fig. 1 Scheme of patients with stage II or III gastric cancer who received standard adjuvant S-1 monotherapy following D2 gastrectomy. $P S$ performance status 
tomography or ultrasonography. Endoscopy was performed 1,3 , and 5 years after the distal gastrectomy.

In this study, the completion of S-1 therapy was defined as $\mathrm{S}-1$ continuing one year to the day after surgery, regardless of the number of courses or total dose. Otherwise the case was regarded as S-1 discontinuation, regardless of the reason.

\section{Staging systems}

Gastric cancer was stage-grouped according to three different systems: the JC-14 (identical to TNM-7) for the primary study purpose, and the JC-13 and TNM- 6 for comparison with the results of the ACTS-GC. Matrixes of these systems are shown in Fig. 2.

\section{Statistical analysis}

Overall survival (OS) was defined as the interval from the date of surgery to the date of death from any cause. Relapsefree survival (RFS) was defined as the interval from surgery to the date of confirmed relapse or death from any cause. The survival rate was estimated using the Kaplan-Meier method. All statistical analyses were performed using the SPSS statistical software ver. 24.0 for Windows.

\section{Results}

\section{Patients}

The clinicopathological features of the 390 patients are shown in Table 1. For comparison, data of the S-1 group of ACTS-GC patients, available from the published papers, are also presented. Age, sex, and type of gastrectomy were very similar between our patients and those of the ACTS-GC. The distribution of pathological stage groups, whether according to the JC-13 or TNM-6, was also similar in the two cohorts but our series included more patients with advanced stages. Some patients in our study with stage IIIB or IIIC according to the JC-14 had stage IV disease according to the JC-13 or TNM-6, mainly because of lymph node metastases judged as N3 (thus stage IV) in the latter systems.

\section{Adjuvant therapy}

Table 2 summarizes the compliance with S-1 monotherapy. The completion rate in our cohort was $69.5 \%$. Within this group, $54.6 \%$ of the patients required a dose reduction and/ or schedule modification. Ten patients started S-1 therapy at our institution but moved to another hospital thereafter, such that S-1 completion could not be determined (although the vital status of the patients was confirmed). Excluding these ten patients, the completion rate was $71.3 \%$. In the
Fig. 2 Matrixes of the staging systems according to JC-14/

TNM-7, and JC-13, and TNM6. JC-14 Japanese Classifications 14th edition, TNM-7 the Union for International Cancer Control 7th edition and American Joint Committee on Cancer 7th edition, $J C-13$ Japanese Classifications 13th edition, TNM-6 the Union for International Cancer Control 6th edition and American Joint Committee on Cancer 6th edition
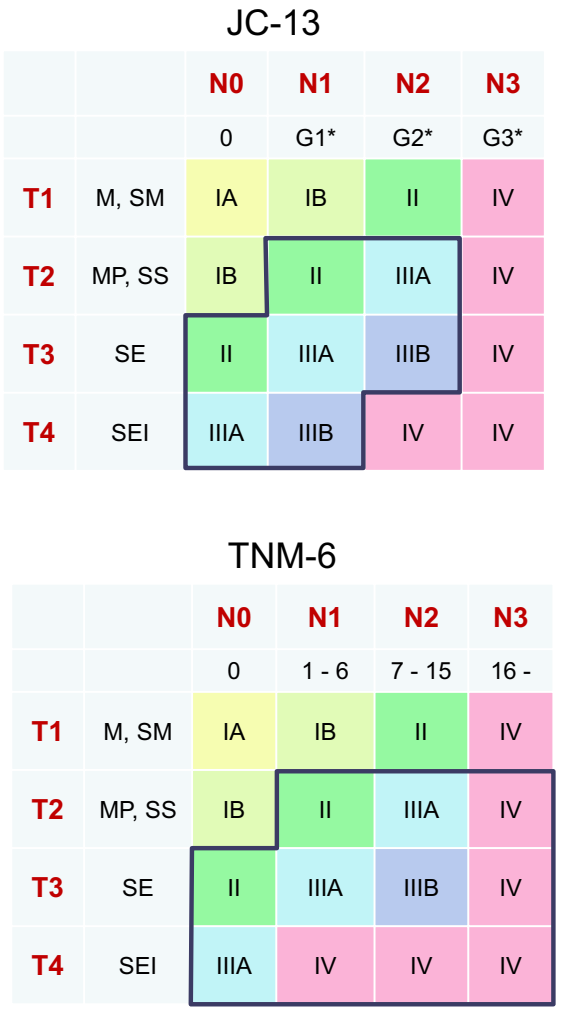

$\mathrm{JC}-14$

TNM-7

\begin{tabular}{|c|c|c|c|c|c|}
\hline & & NO & N1 & N2 & N3 \\
\hline & & 0 & $1-2$ & $3-6$ & $7-$ \\
\hline T1 & $\begin{array}{l}\text { M, } \\
\text { SM }\end{array}$ & IA & IB & IIA & IIB \\
\hline T2 & MP & IB & $\| A$ & IIB & IIIA \\
\hline T3 & SS & IIA & IIB & IIIA & IIIB \\
\hline $\mathrm{T} 4 \mathrm{a}$ & SE & IIB & IIIA & IIIB & IIIC \\
\hline $\mathrm{T} 4 \mathrm{~b}$ & SEI & IIIB & $\mathrm{IIIB}$ & IIIC & IIIC \\
\hline
\end{tabular}

M, mucosa; SM, submucosa; MP, muscularis propria; SE, serosa exposed; SEI, invading adjacent structure

* G1, G2, G3 Groups are determined based on the primary tumor location and the involved lymph node location

Target in this study

Target in ACTS-GC trial 
Table 1 Baseline characteristics of the 390 enrolled patients and those of the ACTS-GC

\begin{tabular}{|c|c|c|}
\hline Characteristic & This study $(n=390)$ & $\begin{array}{l}\text { ACTS-GC S-1 } \\
\text { group }(n=529)\end{array}$ \\
\hline \multicolumn{3}{|l|}{ Sex, $n(\%)$} \\
\hline Male & $270(69.2)$ & $367(69.4)$ \\
\hline Female & $120(30.8)$ & $162(30.6)$ \\
\hline Median age (years old) (range) & $64(27-80)$ & $63(27-80)$ \\
\hline \multicolumn{3}{|l|}{ Type of gastrectomy, $n(\%)$} \\
\hline Total & $165(42.3)$ & $220(41.6)$ \\
\hline Distal & $225(57.7)$ & $301(56.9)$ \\
\hline \multicolumn{3}{|l|}{ Tumor location ${ }^{\mathrm{c}}, n(\%)$} \\
\hline Lower & 109 (27.9) & \\
\hline Middle & $159(40.8)$ & \\
\hline Upper & $109(27.9)$ & \\
\hline Entire & $13(3.3)$ & \\
\hline \multicolumn{3}{|l|}{ Histological type ${ }^{\mathrm{c}}, n(\%)$} \\
\hline Differentiated & $135(34.6)$ & \\
\hline Undifferentiated & $255(65.4)$ & \\
\hline \multicolumn{3}{|l|}{ pT (JC-14/TNM-7), $n(\%)$} \\
\hline $\mathrm{T} 2$ & $60(15.4)$ & $289(54.6)^{\mathrm{a}}$ \\
\hline $\mathrm{T} 3$ & $100(25.7)$ & \\
\hline $\mathrm{T} 4 \mathrm{a}$ & $212(54.4)$ & $225(42.5)$ \\
\hline $\mathrm{T} 4 \mathrm{~b}$ & $18(4.6)$ & $14(2.6)$ \\
\hline \multicolumn{3}{|l|}{ pN (JC-14/TNM-7), $n(\%)$} \\
\hline No & $52(13.3)$ & $51(9.6)$ \\
\hline N1 & $100(25.6)$ & $331(62.6)^{\mathrm{b}}$ \\
\hline $\mathrm{N} 2$ & $107(27.4)$ & \\
\hline N3a & $88(22.6)$ & $117(22.1)$ \\
\hline N3b & $43(11.0)$ & $30(5.7)$ \\
\hline \multicolumn{3}{|l|}{ pStage (JC-14/TNM-7) ${ }^{\mathrm{c}}, n(\%)$} \\
\hline IIA & $25(6.4)$ & \\
\hline IIB & $110(28.2)$ & \\
\hline IIIA & $78(20.0)$ & \\
\hline IIIB & $83(21.3)$ & \\
\hline IIIC & $94(24.1)$ & \\
\hline \multicolumn{3}{|l|}{ pStage (JC-13), $n(\%)$} \\
\hline II & $149(38.2)$ & $236(44.6)$ \\
\hline IIIA & $138(35.4)$ & $202(38.2)$ \\
\hline IIIB & $93(23.8)$ & $90(17.0)$ \\
\hline IV & $10(2.6)$ & $1(0.2)$ \\
\hline \multicolumn{3}{|l|}{ pStage (TNM-6), $n(\%)$} \\
\hline II & $169(43.3)$ & $264(49.9)$ \\
\hline IIIA & $111(28.5)$ & $170(32.1)$ \\
\hline IIIB & $54(13.8)$ & $54(10.2)$ \\
\hline IV & $56(14.4)$ & $40(7.6)$ \\
\hline
\end{tabular}

ACTS-GC Adjuvant Chemotherapy Trial of S-1 for Gastric Cancer, $J C$-14 Japanese Classifications 14th edition, JC-13 Japanese Classifications 13th edition, TNM-7 the Union for International Cancer Control 7th edition and American Joint Committee on Cancer 7th edition, TNM-6 the Union for International Cancer Control 6th edition and American Joint Committee on Cancer 6th edition

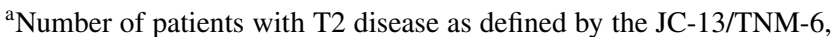
corresponding to T2 and T3 disease as defined by the JC-14/TNM-7

${ }^{\mathrm{b}}$ Number of patients with $\mathrm{N} 1$ as defined by the TNM-6, corresponding to $\mathrm{N} 1$ and $\mathrm{N} 2$ as defined by the JC-14/TNM-7
Table 1 (continued)

${ }^{\mathrm{c}}$ Blank data in ACTS-GC S-1 group were not described in the consulted articles

ACTS-GC, the 12-month compliance was $65.8 \%$, with modifications needed in the treatment of $46.5 \%$ of the patients. Adjuvant S-1 therapy was discontinued in $28.7 \%$ of our patients, most often due to adverse events, followed by disease recurrence. In the ACTS-GC, discontinuation due to treatment refusal was far higher than in our cohort (13.7 vs. $1.8 \%)$.

\section{First relapse site}

During the follow-up period, recurrent disease was confirmed in 115 patients (29.5\%). The first sites of recurrence are listed in Table 3, together with the data reported in the final report of the ACTS-GC [5]. Peritoneal recurrence was the most common, followed by hematogenous and lymph node recurrences. The proportions and patterns of recurrence were very similar between the two cohorts.

\section{Survival}

The vital status of all patients was available at the time of analysis, except for two non-Japanese Asians who returned to their country during follow-up and were thus censored. According to the JC-14/TNM-7, the 3-year RFS rates of patients with pathological stage II and III disease were 89.6 and $69.8 \%$, and those of patients with stage IIA, IIB, IIIA, IIIB, and IIIC disease 100, 87.3, 87.2, 68.7, and 56.4\%, respectively. The 5-year RFS rates of patients with pathological stage II and III disease were 86.7 and $62.4 \%$, and those of patients with stage IIA, IIB, IIIA, IIIB, and IIIC disease $96.0,84.5,78.2,66.3$, and $45.7 \%$, respectively. The 5 -year OS rates of patients with pathological stage II and III disease were 87.3 and $67.3 \%$, and those of patients with stage IIA, IIB, IIIA, IIIB, and IIIC disease 96.0, 85.5, 81.8, 72.0 , and $51.1 \%$, respectively.

The OS and RFS curves are shown in Fig. 3. According to the JC-14/TNM-7, the distance between the stage IIB and IIIB curves was greater for RFS (Fig. 3a) than for OS (Fig. 3b).

Figure $3 \mathrm{c}-\mathrm{f}$ shows the survival curves according to the JC-13 and TNM- 6 classifications. The blue lines represent stage IV disease according to these systems but stage II or III according to the JC-14, and for the most part overlapped with that representing stage IIIB disease.

Table 4 compares the 5-year survival rates of our patients and those of the S-1 group in the ACTS-GC based on the JC-13 and TNM-6 systems. While the inter-substage differences in the 5-year survival rates of the two cohorts 
Table 2 Feasibility of S-1 monotherapy after curative D2 gastrectomy

\begin{tabular}{lcc}
\hline & No. of patients (\%) \\
\cline { 2 - 3 } & This study $(n=390)$ & $\begin{array}{l}\text { ACTS-GC S-1 } \\
\text { group }(n=517)\end{array}$ \\
\hline Total number of completion & $271(69.5)$ & $340(65.8)$ \\
Dose reduction or schedule modifications & $148(37.9)$ & $158(30.5)$ \\
Total number of discontinuations & $109(28.7)$ & $177(34.2)$ \\
Adverse effect & $70(17.9)$ & $72(13.9)$ \\
Recurrence & $32(8.2)$ & $25(4.8)$ \\
Patients' hope or drop out & $7(1.8)$ & $71(13.7)$ \\
Unknown & $10(2.6)$ & \\
\hline
\end{tabular}

ACTS-GC Adjuvant Chemotherapy Trial of S-1 for Gastric Cancer
Table 3 First recurrence site

\begin{tabular}{lcc}
\hline Site & \multicolumn{2}{l}{ No. of patients $(\%)$} \\
\cline { 2 - 3 } & This study $(n=390)$ & $\begin{array}{l}\text { ACTS-GC S-1 } \\
\text { group }(n=529)\end{array}$ \\
\hline Total number of relapses & $115(29.5)$ & $162(30.6)$ \\
Peritoneum & $49(12.6)$ & $77(14.6)$ \\
Hematogenous & $45(11.5)$ & $61(11.5)$ \\
Lymph nodes & $26(6.7)$ & $30(5.7)$ \\
Local & $6(1.5)$ & $11(2.1)$ \\
Unknown & $1(0.3)$ &
\end{tabular}

Some patients had a first recurrence at more than one site ACTS-GC Adjuvant Chemotherapy Trial of S-1 for Gastric Cancer

were similar, our patients had higher survival rates in all substages.

\section{Discussion}

This study provides new information on the recent long-term outcomes of patients who underwent adjuvant S-1 monotherapy after D2 gastrectomy for pathological stage II and III disease as defined by the JC-14/TNM-7. It also provides a first comparison of the survival of patients who received adjuvant S-1 monotherapy at a high-volume center with that of ACTS-GC patients, by applying the same staging systems, i.e., the JC-13 and TNM-6, to the two cohorts.

The survival rates of patients administered adjuvant S-1 monotherapy at our hospital were favorable. The OS curves of those with stage IIB, IIIA, or IIIB disease were very close to each other (Fig. 3b). Even in patients with pathological stage IIIC disease, the 5-year OS was 51.1\%. Future adjuvant or neo-adjuvant therapy using new regimens are expected to exceed these results.

Both OS and RFS rates were higher in our patients than in ACTS-GC patients, in all substages of the JC-13 and TNM-6 (Tables 4). The reasons for this are likely to be multifactorial. First, patient characteristics may be different between the two cohorts. Although the median age of the patients was the same, patients treated in specialized centers in large cities may be physically fitter and more carefully selected with recently available, advanced diagnostic techniques than those treated at general hospitals in the ACTSGC period. Second, stage migration phenomenon should be considered. The proportion of patients with $>15$ metastatic lymph nodes (Table 1, pN3b) was higher in our cohort (11\%) than in the ACTS-GC (5.7\%). This suggests that, at specialized centers, the lymph nodes are more meticulously retrieved from the resected specimen after surgery and are thus more positive nodes are detected, thereby the N-category is up-staged than at general hospitals. This may also be the case for T-category; a larger number of tumor slices per specimens made by pathologists in a specialized center may detect a deeper tumor invasion thereby the T-category is up-staged. This would in most cases result in stage migration and in our patients may account for their better survival across all disease stages. Third, the managements of adjuvant S-1 therapy may have differed, since the 1-year completion rate was higher in our study than in the ACTS-GC, the proportion of dose reduction and/or schedule modifications was higher, and the rate of therapy withdrawal due to treatment refusal was far lower. In the ACTS-GC, S1-therapy was mostly administered by surgeons, at many different hospitals, whereas in our patients it was meticulously managed by medical oncologists at a single specialized center, which may have contributed to a higher completion rate of S-1 adjuvant therapy and thus to better outcomes. Fourth, most of our patients with relapse received a second or even a third line of chemotherapy, which may have prolonged OS. However, because the differences between stage-specific RFS and OS were similar between our patients and those of the ACTS-GC (Tables 4), this assumption may not be valid.

Gastric cancer staging systems underwent an enormous change, from an anatomically based to a numerically based $\mathrm{N}$, between the AJCC/UICC of 1997 and the JGCA of 2010. While earlier classifications (JC-13 or TNM-6) can 
Fig. 3 Kaplan-Meier estimates of RFS (a) and OS (b) according to JC-14//TNM-7, RFS (c) and OS (d) according to JC-13, and RFS (e) and OS (f) according to TNM-6. $R F S$ recurrence-free survival, OS overall survival, $J C$ - 14 Japanese Classifications 14th edition, $T N M-7$ the Union for International Cancer Control 7th edition and American Joint Committee on Cancer 7th edition, JC-13 Japanese Classifications 13th edition, TNM- 6 the Union for International Cancer Control 6th edition and American Joint Committee on Cancer 6th edition a

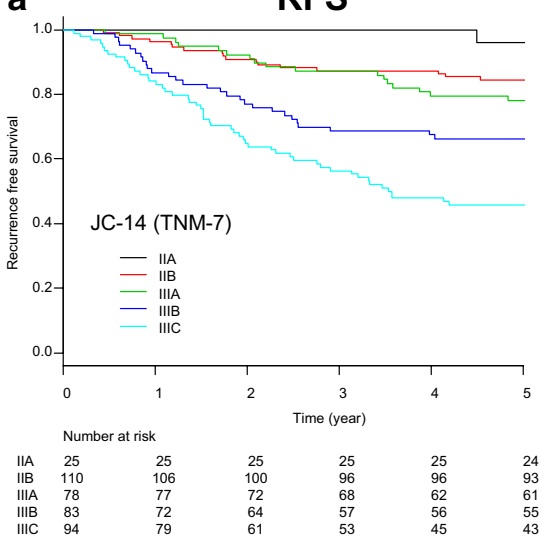

C

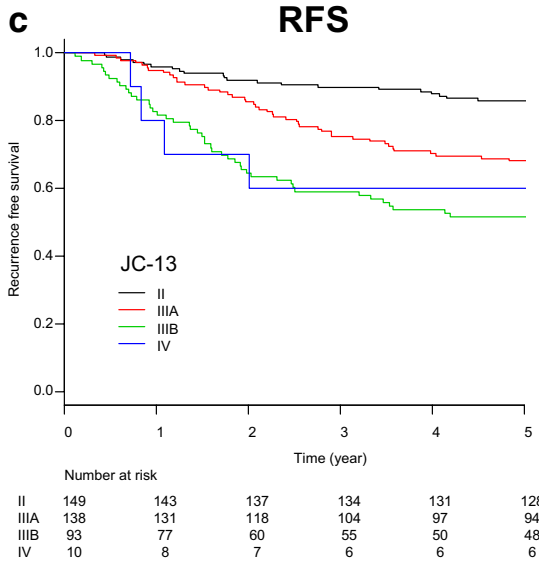

e

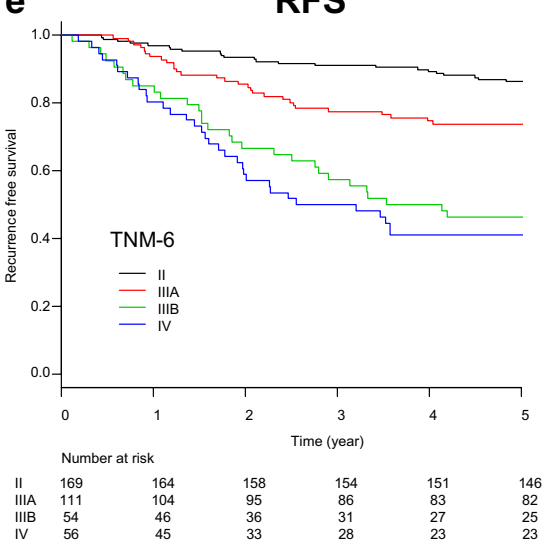

b

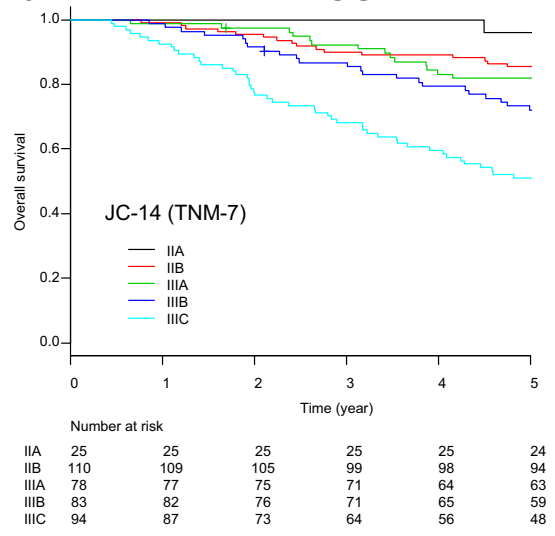

d

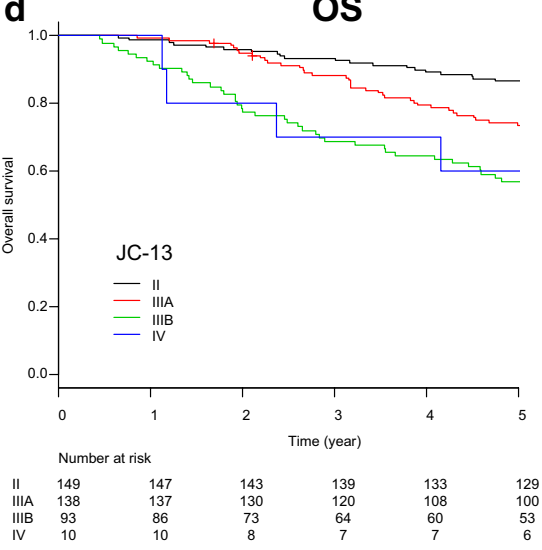

f

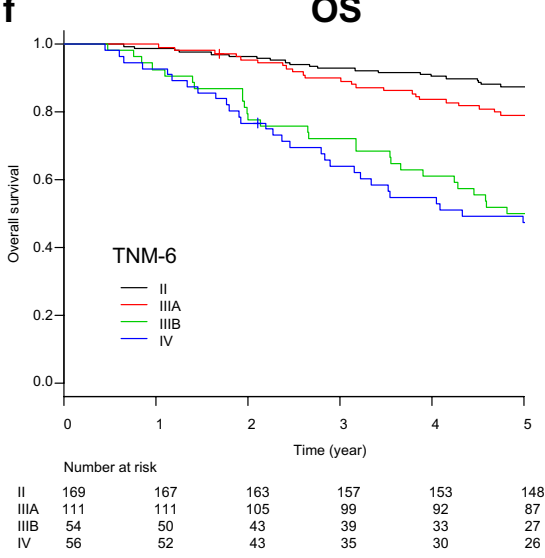

be converted to the new systems (JC-14/TNM-7), the reverse may not be possible because the locations of involved lymph nodes are not always recorded, especially in Western countries. We initially expected that the pathological stages determined in the ACTS-GC, which were recorded using the JC-13, could be easily converted to those of the JC-14/ TNM-7, by accessing the preserved database of the trial. However, this was not possible because, in the multi-institutional ACTS-GC, depth of invasion was recorded only as $\mathrm{T} 2$, $\mathrm{T} 3$, or $\mathrm{T} 4$, in accordance with the $\mathrm{JC}-13$, in which $\mathrm{T} 2$ does not distinguish between invasion of the proper muscle layer and the subserosa. In the JC-14/TNM-7, these findings correspond to $\mathrm{T} 2$ and $\mathrm{T} 3$, respectively, and are independent staging determinants. Thus, the stage-specific survival data in the ACTS-GC cannot be reclassified according to the currently available system. To do so would require re-collecting T2-subcategory information from the records of each trial patient in the 109 hospitals-clearly this is not realistic. Conversely, the conversion of our data describing the current clinical results of S-1 adjuvant therapy, by using the 
Table 4 Comparison of 5-year overall survival and recurrence-free survival in this study and the ACTS-GC according to the JC-13 and TNM-6

\begin{tabular}{|c|c|c|c|c|c|c|c|c|}
\hline \multirow[t]{3}{*}{ Stage } & \multicolumn{4}{|l|}{ RFS } & \multicolumn{4}{|l|}{ OS } \\
\hline & \multicolumn{2}{|l|}{$\mathrm{JC}-13$} & \multicolumn{2}{|l|}{ TNM-6 } & \multicolumn{2}{|l|}{$\mathrm{JC}-13$} & \multicolumn{2}{|l|}{ TNM-6 } \\
\hline & This study (\%) & $\begin{array}{l}\text { ACTS-GC } \\
\text { S-1 group (\%) }\end{array}$ & This study (\%) & $\begin{array}{l}\text { ACTS-GC } \\
\text { S-1 group (\%) }\end{array}$ & This study (\%) & $\begin{array}{l}\text { ACTS-GC } \\
\text { S-1 group (\%) }\end{array}$ & This study (\%) & $\begin{array}{l}\text { ACTS-GC } \\
\text { S-1 group } \\
(\%)\end{array}$ \\
\hline II & 85.9 & 79.2 & 86.4 & 77.9 & 86.6 & 84.2 & 87.6 & 83.4 \\
\hline IIIA & 68.1 & 61.4 & 73.9 & 64.3 & 73.6 & 67.1 & 79.1 & 69.1 \\
\hline IIIB & 51.6 & 37.6 & 46.3 & 35.9 & 57.0 & 50.2 & 50.0 & 44.8 \\
\hline IV & 60.0 & & 41.1 & 26.8 & 60.0 & & 47.1 & 46.0 \\
\hline
\end{tabular}

JC-13 criteria, would enable comparisons with the results of the ACTS-GC but is both difficult and time-consuming. Therefore, the data presented in this study will best serve as a useful reference for future studies.

Shortly after the conclusion of the ACTS-GC, the Japanese Gastric Cancer Treatment Guidelines were revised, such that adjuvant S-1 was newly recommended in the treatment of pathological stage II or III disease [3]. This recommendation was not based on the JC-13 but on the JC-14 published around the same time. Although the definitions of stage II and III are quite different between these two JC editions as mentioned above, it was found that most patients diagnosed as stage II or III by the JC-14, as a whole, correspond to those diagnosed as stage II or III by the JC-13. The only difference is the revised staging of T3N0, which is referred to in the JC-14 as stage IIA but in the JC-13 as stage IB. Thus, the guidelines' recommendation of "adjuvant S-1 for pathological stage II or III except for T3N0" covers most ACTS-GC targets. However, the substage definitions used in the two systems are very different, which should be taken into consideration when the results of adjuvant therapy are compared with those of the ACTS-GC.

In conclusion, survival out come of patients shown in this study will provide a reference for future adjuvant trials targeting JC-14/TNM-7 stage II or III disease. Adjuvant S-1 monotherapy managed by medical oncologists after D2 gastrectomy at a high-volume cancer hospital showed favorable survival as compared to the pivotal trial ACTS-GC. Patient selection, stage migration, and the meticulous managements of S-1 therapy may have contributed to it.

Funding The authors declare that this study had no external funding.

\section{Compliance with ethical standards}

Conflict of interest The authors have no conflicts of interest to declare.

Ethical standards All procedures followed were in accordance with the ethical standards of the institutional and national committees governing human experimentation and in compliance with the Helsinki Declaration of 1964 and later versions.
Informed consent Informed consent or an appropriate substitute was obtained from all patients prior to their inclusion in the study.

\section{References}

1. International Research Agency on Cancer. The GLOBOCAN Project. http://globocan.iarc.fr/Pages/fact_sheets_cancer.aspx. Accessed 1 Dec 2017.

2. National Comprehensive Cancer Network (NCCN). NCCN clinical practice guidelines in oncology. Gastric cancer version 3. 2016.

3. Japanese Gastric Cancer Treatment Guidelines 2014 (ver. 4). Japanese Gastric Cancer Association Gastric Cancer. 2017;20:1-19.

4. Sakuramoto S, Sasako M, Yamaguchi T, Kinoshita T, Fujii M, Nashimoto A, et al. ACTS-GC Group. Adjuvant chemotherapy for gastric cancer with S-1, an oral fluoropyrimidine. N Engl J Med. 2007;357:1810-20.

5. Sasako M, Sakuramoto S, Katai H, Kinoshita T, Furukawa H, Yamaguchi T, et al. Five-year outcomes of a randomized phase III trial comparing adjuvant chemotherapy with $\mathrm{S}-1$ versus surgery alone in stage II or III gastric cancer. J Clin Oncol. 2011;29:4387-93.

6. Japanese Gastric Cancer Association. Japanese classification of gastric carcinoma-2nd English edition. Gastric Cancer 1998;1:10-24.

7. Japanese Gastric Cancer Association. Japanese classification of gastric carcinoma, 13th ed. Tokyo: Kanehara; 1998 (in Japanese).

8. Beahrs OH, Henson DH, Hutter RVP, Kennedy BJ. AJCC cancer staging manual. 4th ed. Philadelphia: Lippincott Company; 1992.

9. Hermanek P, Sobin LH. TNM classification of malignant tumors (UICC), 4th ed. Berlin: Springer; 1987.

10. Fleming ID, Cooper JS, Henson DE, Hutter RVP, Kennedy BJ, Murphy GP. AJCC cancer staging manual, 5th ed. Philadelphia: Lippincott-Raven; 1997.

11. Sobin $\mathrm{LH}$, Wittekind $\mathrm{CH}$. TNM classification of malignant tumors (UICC), 5th ed. New York: Wiley; 1997.

12. Japanese classification of gastric carcinoma: 3rd English edition. Gastric Cancer 2011;14:101-12.

13. Japanese Gastric Cancer Association. Japanese classification of gastric carcinoma, 14th ed. Tokyo: Kanehara; 2010 (in Japanese).

14. Edge SB, Byrd DR, Compton CC, Fritz AG, Greene FL, Trotti A. AJCC cancer staging manual, 7th ed. Chicago: Springer; 2010.

15. Sobin LH, Wittekind C, Gospodarowicz M. TNM classification of malignant tumors (UICC), 7th ed. New York: Wiley; 2009.

16. Greene FL, Page DL, Fleming ID, Fritz A, Balch CM, Haller DG, et al. AJCC cancer staging manual, 6th ed. New York: Springer; 2002.

17. Sobin LH, Wittekind Ch, editors. TNM classification of malignant tumors (UICC), 6th ed. New York: Wiley; 2002. 